\title{
On complete moment convergence for nonstationary negatively associated random variables
}

\section{$\mathrm{Mi}-\mathrm{H} w a \mathrm{Ko}^{*}$}

\section{"Correspondence:}

songhack@wonkwang.ac.kr Division of Mathematics and Informational Statistics, Nanoscale Sciences and Technology Institute, Wonkwang University, Jeonbuk, 570-749, Korea

\section{Springer}

\begin{abstract}
The purpose of this paper is to establish the complete moment convergence for nonstationary negatively associated random variables satisfying the weak mean domination condition. The result is an improvement of complete convergence in Marcinkiewicz-Zygmund-type SLLN for negatively associated random variables in Kuczmaszewska (Acta Math. Hung. 128:116-130, 2010).
\end{abstract}

MSC: 60F15

Keywords: complete moment convergence; negative association; nonstationary; weak mean domination

\section{Introduction}

A sequence of random variables $\left\{X_{n}, n \geq 1\right\}$ is said to converge completely to a constant $c$ if $\sum_{n=1}^{\infty} P\left(\left|X_{n}-c\right|>\epsilon\right)<\infty$ for all $\epsilon>0$. This concept of complete convergence was introduced by Hsu and Robbins [2]. They proved that the sequence of arithmetic means of independent identically distributed random variables converges completely to the expected value of the summands, provided that the variance is finite. The converse theorem was proved by Erdös [3]. This Hsu-Robbins-Erdös's result was generalized in different ways. Katz [4], Baum and Katz [5], and Chow [6] obtained a generalization of complete convergence for a sequence of independent identically distributed random variables with normalization of Marcinkiewicz-Zygmund type (see Gut [7]). Chow [8] first showed the complete moment convergence for a sequence of i.i.d. random variables by generalizing the result of Baum and Katz [5].

The concept of complete moment convergence is as follows. Let $\left\{Y_{n}, n \geq 1\right\}$ be a sequence of random variables, and $a_{n}>0, b_{n}>0, q>0$.

If $\sum_{n=1}^{\infty} a_{n} E\left\{b_{n}^{-1}\left|Y_{n}\right|-\epsilon\right\}_{+}^{q}<\infty$ for all $\epsilon>0$, then $\left\{Y_{n}, n \geq 1\right\}$ is called complete moment convergence. It is well known that the complete moment convergence implies the complete convergence.

Since then, many investigations have been made. For example, Sung [9] obtained a moment inequality for the maximum partial sum of random variables and the complete moment convergence of i.i.d. random variables, Liang and $\mathrm{Li}$ [10] provided necessary and

(c) 2016 Ko. This article is distributed under the terms of the Creative Commons Attribution 4.0 International License (http://creativecommons.org/licenses/by/4.0/), which permits unrestricted use, distribution, and reproduction in any medium, provided you give appropriate credit to the original author(s) and the source, provide a link to the Creative Commons license, and indicate if changes were made. 
sufficient moment conditions for the complete moment convergence of identically distributed negatively associated random variables, and Wu et al. [11] established the complete moment convergence of identically distributed $\rho^{*}$-mixing random variables.

The concept of negative association was introduced by Joag-Dev and Proschan [12] in the following way. A finite family of random variables $\left\{X_{i}, 1 \leq i \leq n\right\}$ is said to be negatively associated if for every pair of disjoint nonempty subsets $A$ and $B$ of $\{1,2, \ldots, n\}$ and any real coordinatewise nondecreasing functions $f$ and $g$,

$$
\operatorname{Cov}\left(f\left(X_{i}, i \in A\right), g\left(X_{j}, j \in B\right)\right) \leq 0
$$

whenever $f$ and $g$ are such that the covariance exists. An infinite family of random variables is negatively associated if every finite subfamily is negatively associated.

A sequence of random variables $\left\{X_{n}, n \geq 1\right\}$ is said to satisfy a weak mean dominating condition with mean dominating random variable $X$ if for some $c>0$,

$$
\frac{1}{n} \sum_{i=1}^{n} P\left(\left|X_{i}\right|>x\right) \leq c P(|X|>x) \quad \text { for all } x>0 \text { and all } n \geq 1 .
$$

Recently, Kuczmaszewska [1] proved the complete convergence for a sequence of negatively associated random variables satisfying (1.2) (see Lemma 2.3).

In this paper, we prove the complete moment convergence for sequences of negatively associated random variables satisfying a weak mean dominating condition. It provides an improvement of complete convergence for negatively associated random variables by Kuczmaszewska [1] and conditions for complete moment convergence for a nonstationary sequence of negatively associated random variables.

\section{Preliminaries}

In our further considerations, we need the following lemmas.

Lemma 2.1 (Shao [13]) Let $\left\{X_{i}, 1 \leq i \leq n\right\}$ be a sequence of negatively associated random variables with $E X_{i}=0$ and $E\left|X_{i}\right|^{q}<\infty$ for $q>1$ and every $1 \leq i \leq n$. Then

$$
E \max _{1 \leq k \leq n}\left|\sum_{i=1}^{k} X_{i}\right|^{q} \leq 2^{3-q} \sum_{i=1}^{n} E\left|X_{i}\right|^{q} \quad \text { for } 1<q \leq 2
$$

and

$$
E \max _{1 \leq k \leq n}\left|\sum_{i=1}^{k} X_{i}\right|^{q} \leq 2\left(\frac{15 q}{\ln q}\right)^{q}\left\{\sum_{i=1}^{n} E\left|X_{i}\right|^{q}+\left(\sum_{i=1}^{n} E X_{i}^{2}\right)^{\frac{q}{2}}\right\} \text { for } q>2 .
$$

Remark In Lemma 2.1 for $q=1$, we have

$$
E \max _{1 \leq k \leq n}\left|\sum_{i=1}^{k} X_{i}\right| \leq \sum_{i=1}^{n} E\left|X_{i}\right|
$$


Lemma 2.2 (Gut [7]) Let $\left\{X_{n}, n \geq 1\right\}$ be a sequence of random variables satisfying a weak mean dominating condition with mean dominating random variable $X$, that is, for some $c>0,(1.2)$ holds for all $x>0$ and $n \geq 1$.

Let $r>0$ and, for some $A>0$,

$$
\begin{aligned}
& X_{i}^{\prime}=X_{i} I\left(\left|X_{i}\right| \leq A\right), \quad X_{i}^{\prime \prime}=X_{i} I\left(\left|X_{i}\right|>A\right), \\
& X_{i}^{*}=X_{i} I\left(\left|X_{i}\right| \leq A\right)-A I\left(X_{i}<-A\right)+A I\left(X_{i}>A\right)
\end{aligned}
$$

and

$$
\begin{aligned}
& X^{\prime}=X I(|X| \leq A), \quad X^{\prime \prime}=X I(|X|>A), \\
& X^{*}=X I(|X| \leq A)-A I(X<-A)+A I(X>A) .
\end{aligned}
$$

Then, for some constant $C>0$,

(i) if $E|X|^{r}<\infty$, then $\left(n^{-1}\right) \sum_{i=1}^{n} E\left|X_{i}\right|^{r} \leq C E|X|^{r}$,

(ii) $\left(n^{-1}\right) \sum_{i=1}^{n} E\left|X_{i}^{\prime}\right|^{r} \leq C\left(E\left|X^{\prime}\right|^{r}+A^{r} P(|X|>A)\right)$ for all $A>0$,

(iii) $\left(n^{-1}\right) \sum_{i=1}^{n} E\left|X_{i}^{\prime \prime}\right|^{r} \leq C E\left|X^{\prime \prime}\right|^{r}$ for all $A>0$,

(iv) $\left(n^{-1}\right) \sum_{i=1}^{n} E\left|X_{i}^{*}\right|^{r} \leq C E\left|X^{*}\right|^{r}$ for all $A>0$.

The following result is obtained by Theorem 2.1 of Kuczmaszewska [1].

Lemma 2.3 (Kuczmaszewska [1]) Let $\alpha p>1, p>0$, and $\alpha>\frac{1}{2}$. Let $\left\{X_{n}, n \geq 1\right\}$ be a sequence of negatively associated random variables with $E X_{n}=0$ for all $n \geq 1$, and $X$ be a random variable possibly defined on a different space satisfying condition (1.2) for all $\epsilon>0$ and $n \geq 1$. Then

$$
E|X|^{p}<\infty
$$

implies

$$
\sum_{n=1}^{\infty} n^{\alpha p-2} P\left(\max _{1 \leq j \leq n}\left|\sum_{i=1}^{j} X_{i}\right|>\epsilon n^{\alpha}\right)<\infty \quad \text { for all } \epsilon>0 .
$$

Lemma 2.4 Let $\alpha p>1, \alpha>\frac{1}{2}$, and $p>0$. Let $\left\{X_{n}, n \geq 1\right\}$ be a sequence of negatively associated random variables with $E X_{n}=0$ for all $n \geq 1$, and $X$ be a random variable possibly defined on a different space satisfying condition (1.2) for all $\epsilon>0$ and $n \geq 1$. Then (2.3) implies

$$
\sum_{n=1}^{\infty} n^{\alpha p-2-\alpha} \int_{n^{\alpha}}^{\infty} P\left(\max _{1 \leq k \leq n}\left|\sum_{i=1}^{k} X_{i}\right|>u\right) d u<\infty .
$$

Proof In the case $0<p<1$, let, for all $u>0$,

$$
X_{i}=X_{i} I\left[\left|X_{i}\right| \leq u\right]+X_{i} I\left[\left|X_{i}\right|>u\right]=X_{u i}+X_{u i}^{\prime}
$$


and

$$
S_{n}=\sum_{i=1}^{n} X_{i} I\left[\left|X_{i}\right| \leq u\right]+\sum_{i=1}^{n} X_{i} I\left[\left|X_{i}\right|>u\right]=\sum_{i=1}^{n} X_{u i}+\sum_{i=1}^{n} X_{u i}^{\prime}
$$

Then we obtain

$$
\begin{aligned}
& \sum_{n=1}^{\infty} n^{\alpha p-2-\alpha} \int_{n^{\alpha}}^{\infty} P\left(\max _{1 \leq j \leq n}\left|S_{j}\right|>u\right) d u \\
& \leq \sum_{n=1}^{\infty} n^{\alpha p-2-\alpha} \int_{n^{\alpha}}^{\infty} P\left(\max _{1 \leq j \leq n}\left|\sum_{i=1}^{j} X_{u i}\right|>\frac{u}{2}\right) d u \\
& \quad+\sum_{n=1}^{\infty} n^{\alpha p-2-\alpha} \int_{n^{\alpha}}^{\infty} P\left(\max _{1 \leq j \leq n}\left|\sum_{i=1}^{j} X_{u i}^{\prime}\right|>\frac{u}{2}\right) d u \\
& :=I+J .
\end{aligned}
$$

For $J$, take $q=p(0<p<1)$. By the Markov inequality, Lemma 2.2(iii), and $E|X|^{p}<\infty$ we have that

$$
\begin{aligned}
J & \leq C \sum_{n=1}^{\infty} n^{\alpha p-2-\alpha} \int_{n^{\alpha}}^{\infty} u^{-\frac{q}{2}} E\left(\max _{1 \leq j \leq n}\left|\sum_{i=1}^{j} X_{u i}^{\prime}\right|\right)^{\frac{q}{2}} d u \text { by Markov inequality } \\
& \leq C \sum_{n=1}^{\infty} n^{\alpha p-2-\alpha} \int_{n^{\alpha}}^{\infty} u^{-\frac{q}{2}} \sum_{i=1}^{n} E\left|X_{u i}^{\prime}\right|^{\frac{q}{2}} d u \quad\left(\frac{q}{2}<\frac{1}{2}\right) \\
& \leq C \sum_{n=1}^{\infty} n^{\alpha p-2-\alpha} \int_{n^{\alpha}}^{\infty} u^{-\frac{q}{2}} \sum_{i=1}^{n} E\left|X_{i}\right|^{\frac{q}{2}} I(|X|>u) d u \\
& \leq C \sum_{n=1}^{\infty} n^{\alpha p-1-\alpha} \int_{n^{\alpha}}^{\infty} u^{-\frac{q}{2}} E|X|^{\frac{q}{2}} I(|X|>u) d u \quad \text { by Lemma 2.2(iii) } \\
& =C \sum_{n=1}^{\infty} n^{\alpha p-1-\alpha} \sum_{m=n}^{\infty} \int_{m^{\alpha}}^{(m+1)^{\alpha}} u^{-\frac{p}{2}} E|X|^{\frac{p}{2}} I(|X|>u) d u \quad(q=p) \\
& \leq C \sum_{n=1}^{\infty} n^{\alpha p-1-\alpha} \sum_{m=n}^{\infty} \int_{m^{\alpha}}^{(m+1)^{\alpha}} m^{-\frac{\alpha p}{2}} E|X|^{\frac{p}{2}} I(|X|>u) d u \\
& \leq C \sum_{m=1}^{\infty} m^{\frac{\alpha p}{2}-1} E|X|^{\frac{p}{2}} I\left(|X|>m^{\alpha}\right) \\
& \leq C \sum_{n=1}^{\infty} n^{\alpha p-1-\alpha} \sum_{m=n}^{\infty} \int_{m^{\alpha}}^{(m+1)^{\alpha}} m^{-\frac{\alpha p}{2}} E|X|^{\frac{p}{2}} I\left(|X|>m^{\alpha}\right) d u \\
& \leq C \sum_{n=1}^{\infty} n^{\alpha p-1-\alpha} \sum_{m=n}^{\infty} m^{-\frac{\alpha p}{2}+\alpha-1} E|X|^{\frac{p}{2}} I\left(|X|>m^{\alpha}\right) \\
& =C|X|^{\frac{p}{2}} I\left(|X|>m^{\alpha}\right) \sum_{n=1}^{m} n^{\alpha p-1-\alpha}
\end{aligned}
$$




$$
\begin{aligned}
& =C \sum_{m=1}^{\infty} m^{\frac{\alpha p}{2}-1} \sum_{n=m}^{\infty} E|X|^{\frac{p}{2}} I\left(n^{\alpha}<|X| \leq(n+1)^{\alpha}\right) \\
& \leq C \sum_{n=1}^{\infty} E|X|^{\frac{p}{2}} I\left(n^{\alpha}<|X| \leq(n+1)^{\alpha}\right) \sum_{m=1}^{n} m^{\frac{\alpha p}{2}-1} \\
& \leq C \sum_{n=1}^{\infty} n^{\frac{\alpha p}{2}} E|X|^{\frac{p}{2}} I\left(n^{\alpha}<|X| \leq(n+1)^{\alpha}\right) \\
& \leq C E|X|^{p}<\infty .
\end{aligned}
$$

Similarly, for $I$, take $q=p(0<p<1)$. By the Markov inequality and Lemma 2.2(ii) we have that

$$
\begin{aligned}
I \leq & C \sum_{n=1}^{\infty} n^{\alpha p-2-\alpha} \int_{n^{\alpha}}^{\infty} u^{-\frac{q}{2}} E\left(\max _{1 \leq i \leq n}\left|\sum_{i=1}^{j} X_{u i}\right|\right)^{\frac{q}{2}} d u \quad \text { by Markov inequality } \\
\leq & C \sum_{n=1}^{\infty} n^{\alpha p-2-\alpha} \int_{n^{\alpha}}^{\infty} u^{-\frac{p}{2}} \sum_{i=1}^{n} E\left|X_{u i}\right|^{\frac{p}{2}} d u \quad\left(q=p \text { and } \frac{p}{2}<\frac{1}{2}\right) \\
\leq & C \sum_{n=1}^{\infty} n^{\alpha p-2-\alpha} \int_{n^{\alpha}}^{\infty} u^{-\frac{p}{2}} \sum_{i=1}^{n}\left\{E\left|X_{i}\right|^{\frac{p}{2}} I\left(\left|X_{i}\right| \leq u\right)\right\} d u \\
\leq & C \sum_{n=1}^{\infty} n^{\alpha p-1-\alpha} \int_{n^{\alpha}}^{\infty} u^{-\frac{p}{2}} E|X|^{\frac{p}{2}} I(|X| \leq u) d u \\
& +C \sum_{n=1}^{\infty} n^{\alpha p-1-\alpha} \int_{n^{\alpha}}^{\infty} P(|X|>u) d u \quad \text { by Lemma 2.2(ii) } \\
\leq & C \sum_{n=1}^{\infty} n^{\alpha p-1-\alpha} \int_{n^{\alpha}}^{\infty} u^{-\frac{p}{2}} E|X|^{\frac{p}{2}} I(|X| \leq u) d u \\
& +C \sum_{n=1}^{\infty} n^{\alpha p-1-\alpha} \int_{n^{\alpha}}^{\infty} u^{-\frac{p}{2}} E|X|^{\frac{p}{2}} I(|X|>u) d u \\
= & I_{1}+I_{2} .
\end{aligned}
$$

In the processing of (2.7), we obtain $I_{2}<\infty$. It remains to prove that $I_{1}<\infty$ :

$$
\begin{aligned}
I_{1} & =C \sum_{n=1}^{\infty} n^{\alpha p-1-\alpha} \sum_{m=n}^{\infty} \int_{m^{\alpha}}^{(m+1)^{\alpha}} u^{-\frac{p}{2}} E|X|^{\frac{p}{2}} I(|X| \leq u) d u \\
& \leq C \sum_{n=1}^{\infty} n^{\alpha p-1-\alpha} \sum_{m=n}^{\infty} m^{-\frac{\alpha p}{2}+\alpha-1} E|X|^{\frac{p}{2}} I\left(|X| \leq(m+1)^{\alpha}\right) \\
& =C \sum_{m=1}^{\infty} m^{-\frac{\alpha p}{2}+\alpha-1} E|X|^{\frac{p}{2}} I\left(|X| \leq(m+1)^{\alpha}\right) \sum_{n=1}^{m} n^{\alpha p-1-\alpha} \\
& \leq C \sum_{m=1}^{\infty} m^{\frac{\alpha p}{2}-1} E|X|^{\frac{p}{2}} I\left(|X| \leq(m+1)^{\alpha}\right) \\
& \leq C \sum_{m=1}^{\infty} m^{\frac{\alpha p}{2}-1} \sum_{n \leq m} E|X|^{\frac{p}{2}} I\left(n^{\alpha}<|X| \leq(n+1)^{\alpha}\right)
\end{aligned}
$$




$$
\begin{aligned}
& \leq C \sum_{m=1}^{\infty} m^{\frac{\alpha p}{2}-1} \sum_{n \leq m} n^{\frac{\alpha p}{2}} P\left(n^{\alpha}<|X| \leq(n+1)^{\alpha}\right) \\
& \leq C \sum_{m=1}^{\infty} m^{\alpha p} P\left(m^{\alpha}<|X| \leq(m+1)^{\alpha}\right) \leq C E|X|^{p}<\infty .
\end{aligned}
$$

Hence, from (2.6)-(2.8) the result (2.5) follows in the case $0<p<1$.

In the case $1 \leq p<2$, let $Y_{u i}=X_{i} I\left(\left|X_{i}\right| \leq u\right)-u I\left(X_{i}<-u\right)+u I\left(X_{i}>u\right)$ for all $u>0$, and $Y_{u i}^{\prime}=X_{i}-Y_{u i}, i \geq 1$. Then we have

$$
\begin{aligned}
& \sum_{n=1}^{\infty} n^{\alpha p-2-\alpha} \int_{n^{\alpha}}^{\infty} P\left(\max _{1 \leq k \leq n}\left|\sum_{i=1}^{k} X_{i}\right|>u\right) d u \\
& \leq \sum_{n=1}^{\infty} n^{\alpha p-2-\alpha} \int_{n^{\alpha}}^{\infty} P\left(\max _{1 \leq k \leq n}\left|\sum_{i=1}^{k}\left(Y_{u i}-E Y_{u i}\right)\right|>\frac{u}{2}\right) d u \\
& \quad+\sum_{n=1}^{\infty} n^{\alpha p-2-\alpha} \int_{n^{\alpha}}^{\infty} P\left(\max _{1 \leq k \leq n}\left|\sum_{i=1}^{k}\left(Y_{u i}^{\prime}-E Y_{u i}^{\prime}\right)\right|>\frac{u}{2}\right) d u \text { since } E X_{n}=0 \\
& :=I^{\prime}+J^{\prime} .
\end{aligned}
$$

Note that $\left\{Y_{u i}-E Y_{u i}\right\}$ and $\left\{Y_{u i}^{\prime}-E Y_{u i}^{\prime}\right\}$ are sequences of negatively associated random variables.

For $J^{\prime}$, take $q$ such that $1 \leq p<q \leq 2$. By the fact that $\left|Y_{u i}^{\prime}\right| \leq\left|X_{i}\right| I\left(\left|X_{i}\right|>u\right)$, the Markov inequality, Lemma 2.1, (2.1), Lemma 2.2(iii), (2.3), and the $C_{r}$-inequality we have

$$
\begin{aligned}
J^{\prime} & \leq C \sum_{n=1}^{\infty} n^{\alpha p-2-\alpha} \int_{n^{\alpha}}^{\infty} u^{-q} \sum_{i=1}^{n} E\left|Y_{u i}^{\prime}-E Y_{u i}^{\prime}\right|^{q} d u \quad \text { by (2.1) } \\
& \leq C \sum_{n=1}^{\infty} n^{\alpha p-2-\alpha} \int_{n^{\alpha}}^{\infty} u^{-q} \sum_{i=1}^{n} E\left|Y_{u i}^{\prime}\right|^{q} d u \quad \text { (by the } C_{r} \text {-inequality) } \\
& \leq C \sum_{n=1}^{\infty} n^{\alpha p-2-\alpha} \int_{n^{\alpha}}^{\infty} u^{-q} \sum_{i=1}^{n} E\left|X_{i}\right|^{q} I\left(\left|X_{i}\right|>u\right) d u \\
& \leq C \sum_{n=1}^{\infty} n^{\alpha p-1-\alpha} \int_{n^{\alpha}}^{\infty} u^{-q} E|X|^{q} I(|X|>u) d u \quad \text { Lemma 2.2(iii) } \\
& =C \sum_{n=1}^{\infty} n^{\alpha p-1-\alpha} \sum_{m=n}^{\infty} \int_{m^{\alpha}}^{(m+1)^{\alpha}} u^{-q} E|X|^{q} I(|X|>u) d u \\
& \leq C \sum_{n=1}^{\infty} n^{\alpha p-1-\alpha} \sum_{m=n}^{\infty} m^{-\alpha q+\alpha-1} E|X|^{q} I\left(|X|>m^{\alpha}\right) \\
& \leq C \sum_{m=1}^{\infty} m^{-\alpha q+\alpha-1} E|X|^{q} I\left(|X|>m^{\alpha}\right) \sum_{n=1}^{m} n^{\alpha p-1-\alpha} \\
& =C \sum_{m=1}^{\infty} m^{-\alpha(q-p)-1} E|X|^{q} I\left(|X|>m^{\alpha}\right) \\
& =C \sum_{m=1}^{\infty} m^{-\alpha(q-p)-1} \sum_{n=m}^{\infty} E|X|^{q} I\left(n^{\alpha}<|X| \leq(n+1)^{\alpha}\right)
\end{aligned}
$$




$$
\begin{aligned}
& \leq C \sum_{n=1}^{\infty} E|X|^{q} I\left(n^{\alpha}<|X| \leq(n+1)^{\alpha}\right) \sum_{m=1}^{n} m^{-\alpha(q-p)-1} \\
& \leq C \sum_{n=1}^{\infty} E|X|^{p} I\left(n^{\alpha}<|X| \leq(n+1)^{\alpha}\right) \\
& \leq C E|X|^{p}<\infty .
\end{aligned}
$$

For $I^{\prime}$, take $q$ such that $1<p<q \leq 2$. By the Markov inequality, (1.2), Lemma 2.1, (2.1), the $C_{r}$-inequality, and Lemma 2.2(iv) we have

$$
\begin{aligned}
I^{\prime} \leq & C \sum_{n=1}^{\infty} n^{\alpha p-2-\alpha} \int_{n^{\alpha}}^{\infty} u^{-q} E\left(\max _{1 \leq k \leq n} \sum_{i=1}^{k}\left|Y_{u i}-E Y_{u i}\right|^{q}\right) d u \quad \text { by Markov inequality } \\
\leq & C \sum_{n=1}^{\infty} n^{\alpha p-2-\alpha} \int_{n^{\alpha}}^{\infty} u^{-q} \sum_{i=1}^{n} E\left|Y_{u i}-E Y_{u i}\right|^{q} d u \quad \text { by }(2.1) \\
\leq & C \sum_{n=1}^{\infty} n^{\alpha p-2-\alpha} \int_{n^{\alpha}}^{\infty} u^{-q} \sum_{i=1}^{n} E\left|Y_{u i}\right|^{q} d u \quad \text { by the } C_{r} \text {-inequality } \\
\leq & C \sum_{n=1}^{\infty} n^{\alpha p-1-\alpha} \int_{n^{\alpha}}^{\infty} u^{-q} E|X|^{q} I(|X| \leq u) d u \\
& +C \sum_{n=1}^{\infty} n^{\alpha p-1-\alpha} \int_{n^{\alpha}}^{\infty} u^{-q} E|X|^{q} I(|X|>u) d u \quad \text { by Lemma 2.2(iv) } \\
= & I_{1}^{\prime}+I_{2}^{\prime} .
\end{aligned}
$$

According to the calculation of $J^{\prime}$, we obtain $I_{2}^{\prime}<\infty$ (see (2.10)). It remains to prove that $I_{1}^{\prime}<\infty$. By taking $q$ such that $1 \leq p<q \leq 2$ we have

$$
\begin{aligned}
I_{1}^{\prime} & =C \sum_{n=1}^{\infty} n^{\alpha p-1-\alpha} \sum_{m=n}^{\infty} \int_{m^{\alpha}}^{(m+1)^{\alpha}} u^{-q} E|X|^{q} I(|X| \leq u) d u \\
& \leq C \sum_{n=1}^{\infty} n^{\alpha p-1-\alpha} \sum_{m=n}^{\infty} m^{-\alpha q+\alpha-1} E|X|^{q} I\left(|X| \leq(m+1)^{\alpha}\right) \\
& =C \sum_{m=1}^{\infty} m^{-\alpha q+\alpha-1} E|X|^{q} I\left(|X| \leq(m+1)^{\alpha}\right) \sum_{n=1}^{m} n^{\alpha p-1-\alpha} \\
& \leq C \sum_{m=1}^{\infty} m^{-\alpha q+\alpha p-1} E|X|^{q} I\left(|X| \leq(m+1)^{\alpha}\right) \\
& \leq C \sum_{m=1}^{\infty} m^{-\alpha q+\alpha p-1} \sum_{n \leq m} E|X|^{q} I\left(n^{\alpha}<|X| \leq(n+1)^{\alpha}\right) \\
& \leq C \sum_{m=1}^{\infty} m^{\alpha p} P\left(m^{\alpha}<|X| \leq(m+1)^{\alpha}\right) \\
& =C E|X|^{p}<\infty .
\end{aligned}
$$

Hence, from (2.9)-(2.11) the result (2.5) follows in the case $1 \leq p<2$.

In the case $p \geq 2$, we also obtain $I^{\prime}$ and $J^{\prime}$ of (2.9). 
For $I^{\prime}$, by the Markov inequality, the $C_{r}$-inequality, and Lemma 2.1 (2.2) we have that, for $q>2$,

$$
\begin{aligned}
I^{\prime} \leq & C \sum_{n=1}^{\infty} n^{\alpha p-2-\alpha} \int_{n^{\alpha}}^{\infty} u^{-q} E\left\{\max _{1 \leq j \leq n}\left|\sum_{i=1}^{j}\left(Y_{u i}-E Y_{u i}\right)\right|\right\}^{q} d u \\
\leq & C \sum_{n=1}^{\infty} n^{\alpha p-2-\alpha} \int_{n^{\alpha}}^{\infty} u^{-q}\left\{\sum_{i=1}^{n} E\left|Y_{u i}-E Y_{u i}\right|^{q}+\left(\sum_{i=1}^{n} E\left|Y_{u i}-E Y_{u i}\right|^{2}\right)^{\frac{q}{2}}\right\} d u \quad \text { by }(2.2) \\
\leq & C \sum_{n=1}^{\infty} n^{\alpha p-2-\alpha} \int_{n^{\alpha}}^{\infty} u^{-q} \sum_{i=1}^{n} E\left|Y_{u i}\right|^{q} d u \\
& +C \sum_{n=1}^{\infty} n^{\alpha p-2-\alpha} \int_{n^{\alpha}}^{\infty} u^{-q}\left(\sum_{i=1}^{n} E Y_{u i}^{2}\right)^{\frac{q}{2}} d u \quad \text { by the } C_{r} \text {-inequality } \\
= & I_{3}^{\prime}+I_{4}^{\prime} .
\end{aligned}
$$

We will consider $I_{3}^{\prime}$ and $I_{4}^{\prime}$ as follows.

Note that $\alpha>\frac{1}{2}, \alpha p>1$, and $p \geq 2$. Take $q>\max \left(p, \frac{\alpha p-1}{\alpha-\frac{1}{2}}\right)$, which implies that $\alpha p-2-$ $\alpha q+\frac{q}{2}<-1$.

By Lemma 2.2(iv) we have

$$
\begin{aligned}
I_{3}^{\prime} \leq & C \sum_{n=1}^{\infty} n^{\alpha p-1-\alpha} \int_{n^{\alpha}}^{\infty} u^{-q} E|X|^{q} I(|X| \leq u) d u \\
& +C \sum_{n=1}^{\infty} n^{\alpha p-1-\alpha} \int_{n^{\alpha}}^{\infty} u^{-q} E|X|^{q} I(|X|>u) d u \\
\leq & I_{31}^{\prime}+I_{32}^{\prime} .
\end{aligned}
$$

According to the calculation of $J^{\prime}$, we obtain $I_{32}^{\prime}<\infty$ (see (2.10)). It remains to prove $I_{31}^{\prime}$ :

$$
\begin{aligned}
I_{31}^{\prime} \leq & C \sum_{n=1}^{\infty} n^{\alpha p-1-\alpha} \sum_{m=n}^{\infty} \int_{m^{\alpha}}^{(m+1)^{\alpha}} u^{-q} E|X|^{q} I(|X| \leq u) d u \\
\leq & C \sum_{n=1}^{\infty} n^{\alpha p-1-\alpha} \sum_{m=n}^{\infty} m^{\alpha-1-\alpha q} E|X|^{q} I\left(|X| \leq(m+1)^{\alpha}\right) \\
= & C \sum_{m=1}^{\infty} m^{\alpha-1-\alpha q} E|X|^{q} I\left(|X| \leq(m+1)^{\alpha}\right) \sum_{m=1}^{n} m^{\alpha p-1-\alpha} \\
\leq & C \sum_{n=1}^{\infty} n^{\alpha p-1-\alpha q} E|X|^{q} I\left(|X| \leq(m+1)^{\alpha}\right) \\
\leq & C \sum_{m=1}^{\infty} m^{\alpha p-1-\alpha q} E|X|^{q} I\left(m^{\alpha}<|X| \leq(m+1)^{\alpha}\right) \\
& +C \sum_{m=1}^{\infty} m^{\alpha p-1-\alpha q} E|X|^{q} I\left(|X| \leq m^{\alpha}\right)
\end{aligned}
$$




$$
\begin{aligned}
\leq & C \sum_{m=1}^{\infty} m^{-1} E|X|^{p} I\left(m^{\alpha}<|X| \leq(m+1)^{\alpha}\right) \\
& +C \sum_{m=1}^{\infty} m^{-\alpha(q-p)-1} \sum_{j=1}^{m} j^{\alpha q} P\left((j-1)^{\alpha}<|X| \leq j^{\alpha}\right) \\
\leq & C \sum_{m=1}^{\infty} E|X|^{p} I\left(m^{\alpha}<|X| \leq(m+1)^{\alpha}\right) \\
& +C \sum_{j=1}^{\infty} j^{\alpha q} P\left((j-1)^{\alpha}<|X| \leq j^{\alpha}\right) \sum_{m=j}^{\infty} m^{-\alpha(q-p)-1} \\
\leq & C E|X|^{p}+C \sum_{j=1}^{\infty} j^{\alpha p} P\left((j-1)^{\alpha}<|X| \leq j^{\alpha}\right) \\
\leq & C E|X|^{p}<\infty,
\end{aligned}
$$

which yields $I_{3}^{\prime}<\infty$.

By the fact that $E X^{2}<\infty$ and $E|X|^{p}<\infty$ we have for $p \geq 2$ :

$$
\begin{aligned}
I_{4}^{\prime} & \leq C \sum_{n=1}^{\infty} n^{\alpha p-2-\alpha} \int_{n^{\alpha}}^{\infty} u^{-q}\left(\sum_{i=1}^{n} E X_{i}^{2}\right)^{\frac{q}{2}} d u \\
& \leq C \sum_{n=1}^{\infty} n^{\alpha p-2-\alpha} \int_{n^{\alpha}}^{\infty} u^{-q}\left(n E X^{2}\right)^{\frac{q}{2}} d u \\
& \leq C \sum_{n=1}^{\infty} n^{\alpha p-2-\alpha+\frac{q}{2}} \int_{n^{\alpha}}^{\infty} u^{-q} d u \\
& \leq C \sum_{n=1}^{\infty} n^{\alpha p-2-\alpha q+\frac{q}{2}}<\infty .
\end{aligned}
$$

Thus, the proof of Lemma 2.4 is complete.

\section{Main results}

Theorem 3.1 Let $\alpha p>1, p>0$, and $\alpha>\frac{1}{2}$. Let $\left\{X_{n}, n \geq 1\right\}$ be a sequence of negatively associated random variables with $E X_{n}=0$ for all $n \geq 1$, and $X$ be a random variable satisfying conditions (1.2) and $E|X|^{p}<\infty$. Then, for all $\epsilon>0$,

$$
\sum_{n=1}^{\infty} n^{\alpha p-2-\alpha} E\left(\max _{1 \leq k \leq n}\left|\sum_{i=1}^{k} X_{i}\right|-\epsilon n^{\alpha}\right)^{+}<\infty,
$$

where $a^{+}=\max \{a, 0\}$.

Proof To prove (3.1), we use Lemmas 2.3 and 2.4 as follows:

$$
\begin{aligned}
& \sum_{n=1}^{\infty} n^{\alpha p-2-\alpha} E\left(\max _{1 \leq k \leq n}\left|\sum_{i=1}^{k} X_{i}\right|-\epsilon n^{\alpha}\right)^{+} \\
& \quad=\sum_{n=1}^{\infty} n^{\alpha p-2-\alpha} \int_{0}^{\infty} P\left(\left(\max _{1 \leq k \leq n}\left|\sum_{i=1}^{k} X_{i}\right|-\epsilon n^{\alpha}\right)^{+}>u\right) d u
\end{aligned}
$$




$$
\begin{aligned}
= & \sum_{n=1}^{\infty} n^{\alpha p-2-\alpha} \int_{0}^{\infty} P\left(\max _{1 \leq k \leq n}\left|\sum_{i=1}^{k} X_{i}\right|-\epsilon n^{\alpha}>u\right) d u \\
= & \sum_{n=1}^{\infty} n^{\alpha p-2-\alpha} \int_{0}^{n^{\alpha}} P\left(\max _{1 \leq k \leq n}\left|\sum_{i=1}^{k} X_{i}\right|-\epsilon n^{\alpha}>u\right) d u \\
& +\sum_{n=1}^{\infty} n^{\alpha p-2-\alpha} \int_{n^{\alpha}}^{\infty} P\left(\max _{1 \leq k \leq n}\left|\sum_{i=1}^{k} X_{i}\right|-\epsilon n^{\alpha}>u\right) d u \\
\leq & \sum_{n=1}^{\infty} n^{\alpha p-2} P\left(\max _{1 \leq k \leq n}\left|\sum_{i=1}^{k} X_{i}\right|>\epsilon n^{\alpha}\right) \\
& +\sum_{n=1}^{\infty} n^{\alpha p-2-\alpha} \int_{n^{\alpha}}^{\infty} P\left(\max _{1 \leq k \leq n}\left|\sum_{i=1}^{k} X_{i}\right|>u\right) d u .
\end{aligned}
$$

By Lemma 2.3 the first term of (3.2) is finite, and by Lemma 2.4 the second term of (3.2) is finite. Hence, the proof of the theorem is complete.

Theorem 3.2 Let $\alpha p>1, p>0$, and $\alpha>\frac{1}{2}$. Let $\left\{X_{n}, n \geq 1\right\}$ be a sequence of negatively associated random variables with $E X_{n}=0$ for all $n \geq 1$, and $X$ be a random variable possibly defined on a different space satisfying the condition (1.2). Then (3.1) implies (2.4).

Proof It is easy to see that

$$
\begin{aligned}
& \sum_{n=1}^{\infty} n^{\alpha p-2-\alpha} E\left(\max _{1 \leq k \leq n}\left|\sum_{i=1}^{k} X_{i}\right|-\epsilon n^{\alpha}\right)^{+} \\
& \geq \sum_{n=1}^{\infty} n^{\alpha p-2-\alpha} \int_{0}^{\infty} P\left(\max _{1 \leq k \leq n}\left|\sum_{i=1}^{k} X_{i}\right|-\epsilon n^{\alpha}>u\right) d u \\
& \geq \sum_{n=1}^{\infty} n^{\alpha p-2-\alpha} \int_{0}^{\epsilon n^{\alpha}} P\left(\max _{1 \leq k \leq n}\left|\sum_{i=1}^{k} X_{i}\right|>\epsilon n^{\alpha}+u\right) d u \\
& \geq \epsilon \sum_{n=1}^{\infty} n^{\alpha p-2} P\left(\max _{1 \leq k \leq n}\left|\sum_{i=1}^{k} X_{i}\right|>2 \epsilon n^{\alpha}\right) .
\end{aligned}
$$

Hence, by (3.3), (3.1) implies (2.4). The proof of the theorem is complete.

Remark According to Ko [14], the results on the complete moment convergence for negatively associated random variables was established only in the case $p>1$. In this paper, it is obtained in the case $p>0$.

Theorem 3.3 Let $\alpha p>1, p>0$, and $\alpha>\frac{1}{2}$. Let $\left\{X_{n}, n \geq 1\right\}$ be a sequence of negatively associated random variables with $E X_{n}=0$ for all $n \geq 1$, and $X$ be a random variable satisfying condition (1.2) and $E|X|^{p}<\infty$. Then, for all $\epsilon>0$,

$$
\sum_{n=1}^{\infty} n^{\alpha p-2} E\left\{\sup _{k \geq n}\left|k^{-\alpha} \sum_{j=1}^{k} X_{j}\right|-\epsilon\right\}^{+}<\infty .
$$


Proof By (3.1) we have

$$
\begin{aligned}
& \sum_{n=1}^{\infty} n^{\alpha p-2} E\left\{\sup _{k \geq n}\left|k^{-\alpha} \sum_{i=1}^{k} X_{i}\right|-\epsilon\right\}^{+} \\
& =\sum_{n=1}^{\infty} n^{\alpha p-2} \int_{0}^{\infty} P\left(\sup _{k \geq n}\left|k^{-\alpha} \sum_{i=1}^{k} X_{i}\right|>\epsilon+u\right) d u \\
& =\sum_{j=1}^{\infty} \sum_{n=2^{j-1}}^{2^{j}-1} n^{\alpha p-2} \int_{0}^{\infty} P\left(\sup _{k \geq n}\left|k^{-\alpha} \sum_{i=1}^{k} X_{i}\right|>\epsilon+u\right) d u \\
& \leq C \sum_{j=1}^{\infty} \int_{0}^{\infty} P\left(\sup _{k \geq 2^{j-1}}\left|k^{-\alpha} \sum_{i=1}^{k} X_{i}\right|>\epsilon+u\right) d u \sum_{n=2^{j-1}}^{2^{j}-1} n^{\alpha p-2} \\
& \leq C \sum_{j=1}^{\infty} 2^{j(\alpha p-1)} \int_{0}^{\infty} P\left(\sup _{k \geq 2^{j-1}}\left|k^{-\alpha} \sum_{i=1}^{k} X_{i}\right|>\epsilon+u\right) d u \\
& \leq C \sum_{j=1}^{\infty} 2^{j(\alpha p-1)} \sum_{m=j}^{\infty} \int_{0}^{\infty} P\left(\max _{2^{m}-1 \leq k \leq 2^{m}}\left|k^{-\alpha} \sum_{i=1}^{k} X_{i}\right|>\epsilon+u\right) d u \\
& \leq C \sum_{m=1}^{\infty} \int_{0}^{\infty} P\left(\max _{2^{m}-1 \leq k \geq 2^{m}}\left|k^{-\alpha} \sum_{i=1}^{k} X_{i}\right|>\epsilon+u\right) d u \sum_{j=1}^{m} 2^{j(\alpha p-1)} \\
& \leq C \sum_{m=1}^{\infty} 2^{m(\alpha p-1)} \int_{0}^{\infty} P\left\{\max _{2^{m}-1 \leq k \leq 2^{m}}\left|\sum_{i=1}^{k} X_{i}\right|>(\epsilon+u) 2^{(m-1) \alpha}\right\} d u \\
& \text { (letting } \left.y=2^{(m-1) \alpha} u\right) \\
& \leq C \sum_{m=1}^{\infty} 2^{m(\alpha p-1-\alpha)} \int_{0}^{\infty} P\left(\max _{1 \leq k \leq 2^{m}}\left|\sum_{i=1}^{k} X_{i}\right|>\epsilon 2^{(m-1) \alpha}+y\right) d y \\
& \leq C \sum_{n=1}^{\infty} n^{\alpha p-2-\alpha} \int_{0}^{\infty} P\left(\max _{1 \leq k \leq n}\left|\sum_{i=1}^{k} X_{i}\right|>\epsilon n^{\alpha} 2^{-\alpha}+y\right) d y \\
& =C \sum_{n=1}^{\infty} n^{\alpha p-2-\alpha} E\left(\max _{1 \leq k \leq n}\left|\sum_{i=1}^{k} X_{i}\right|-\epsilon^{\prime} n^{\alpha}\right)^{+}<\infty \text {, }
\end{aligned}
$$

where $\epsilon^{\prime}=\epsilon 2^{-\alpha}$. Hence, the proof of (3.4) is completed.

Theorem 3.4 Let $\alpha>\frac{1}{2}, p>0$, and $\alpha p>1$. Let $\left\{X_{n}, n \geq 1\right\}$ be a sequence of negatively associated random variables with $E X_{n}=0$ for all $n \geq 1$, and $X$ be a random variable satisfying condition (1.2) with $E|X|^{p}<\infty$. Then

$$
\sum_{n=1}^{\infty} n^{\alpha p-2} P\left(\sup _{j \geq n}\left|\frac{S_{j}}{j^{\alpha}}\right| \geq \epsilon\right)<\infty
$$

for all $\epsilon>0$.

Proof Inspired by the proof of Theorem 12.1 of Gut [15], we can prove it and omit the proof. 


\section{Competing interests}

The author declares that there is no conflict of interests regarding the publication of this article.

\section{Acknowledgements}

The author is exceptionally grateful to the referee for offering helpful comments and suggestions. This paper was supported by Wonkwang University in 2016.

Received: 28 August 2015 Accepted: 26 April 2016 Published online: 04 May 2016

\section{References}

1. Kuczmaszewska, A: On complete convergence in Marcinkiewicz-Zygmund type SLLN for negatively associated random variables. Acta Math. Hung. 28, 116-130 (2010)

2. Hsu, PL, Robbins, H: Complete convergence and the law of large numbers. Proc. Natl. Acad. Sci. USA 33(2), 25-31 (1947)

3. Erdös, P: On a theorem of Hsu and Robbins. Ann. Math. Stat. 20(2), 286-291 (1949)

4. Katz, ML: The probability in the tail of a distribution. Ann. Math. Stat. 34, 312-318 (1963)

5. Baum, LE, Katz, M: Convergence rates in the law of large numbers. Trans. Am. Math. Soc. 120(1), 108-123 (1965)

6. Chow, YS: Delayed sums and Borel summability of independent, identically distributed random variables. Bull. Inst. Math. Acad. Sin. 1, 207-220 (1973)

7. Gut, A: Complete convergence for arrays. Period. Math. Hung. 25, 51-75 (1992)

8. Chow, YS: On the rate of moment complete convergence of sample sums and extremes. Bull. Inst. Math. Acad. Sin. $16,177-2011(1988)$

9. Sung, SH: Moment inequalities and complete moment convergence. J. Inequal. Appl. 2009, 271265 (2009)

10. Liang, HY, Li, DL: Complete moment and integral convergence for sums of negatively associated random variables. Acta Math. Sin. Engl. Ser. 26(3), 419-432 (2010)

11. Wu, YF, Wang, $\mathrm{CH}$, Volodin, A: Limiting behavior for arrays of rowwise $\rho^{*}$-mixing random variables. Lith. Math. J. 52(2), 214-221 (2012)

12. Joag-Dev, K, Proschan, F: Negative association of random variables with applications. Ann. Stat. 11, $286-295$ (1983)

13. Shao, QM: A comparison theorem on moment inequalities between negatively associated and independent random variables. J. Theor. Probab. 13, 343-356 (2000)

14. Ko, M-H: Complete moment convergence of moving average process generated by a class of random variables. J. Inequal. Appl. (2015). doi:10.1186/s13660-015-0745-x

15. Gut, A: Probability: A Graduate Course. Springer, New York (2005)

\section{Submit your manuscript to a SpringerOpen ${ }^{\circ}$ journal and benefit from:}

- Convenient online submission

Rigorous peer review

- Immediate publication on acceptance

- Open access: articles freely available online

- High visibility within the field

- Retaining the copyright to your article 